\title{
Optimización de las Condiciones de Operación de la Micro- gasificación de Biomasa para Producción de Gas de Síntesis
}

\author{
Luz M. Ahumada, Arnaldo Verdeza, Antonio J. Bula y Jahir Lombana \\ Facultad de Ingeniería, Dpto. de Ingeniería Mecánica, Univ. del Norte, Barranquilla-Colombia \\ (e-mail: Imahumada@Uninorte.edu.co; averdeza@uninorte.edu.co; abula@uninorte.edu.co; \\ lombanaj@uninorte.edu.co)
}

Recibido Sep. 25, 2015; Aceptado Dic. 1, 2015; Versión final Ene. 18, 2016, Publicado Jun. 2016

\begin{abstract}
Resumen
Se presentan los resultados de un estudio sobre la significancia y la optimización de algunas variables (granulometría, velocidad de aire, contenido de biomasa y disposición del reactor) en el poder calorífico del gas de síntesis obtenido de la gasificación de biomasa (carbón vegetal y cuesco de palma africana). Mediante un diseño de experimento se evaluaron las cuatro variables que oscilaban entre $8-13 \mathrm{~mm}$ para la granulometría, $0.8-1.4 \mathrm{~m} / \mathrm{s}$ para la velocidad del aire, 0-100 para el contenido de biomasa y ascendentedescendente para la disposición del reactor. Se encontró que los factores correspondientes a la granulometría y el contenido de biomasa resultan ser los más significativos en el poder calorífico del gas. Un poder calorífico máximo de $3.84 \mathrm{MJ} / \mathrm{Nm}^{3}$ se obtuvo con la disposición descendente del reactor, alimentación de carbón vegetal con granulometría de $13 \mathrm{~mm}$ y suministro máximo de flujo de aire. La verificación del punto óptimo de operación mostró que tales condiciones de operación favorecían la producción de un gas con un alto poder calorífico.
\end{abstract}

Palabras clave: gasificación de biomasa; poder calorífico; conversión termoquímica; optimización; gas de síntesis

\section{Operating Condition Optimization of Biomass Micro- gasification for Syngas Production}

\begin{abstract}
The results of study on the significance and the optimization of some variables (particle size, air flow, biomass content and reactor disposition) in the calorific value of the synthesis gas obtained by gasification process of biomass (vegetal coal and biomass African palm) is presented. The four variables ranged from $8-13 \mathrm{~mm}$ for the particle size, $0.8-1.4 \mathrm{~m} / \mathrm{s}$ for air flow, $0-100$ for biomass content and updraft-downdraft disposition for the type of reactor were evaluated by an experimental design. It was found that the particle size and biomass content are the most significant factors to maximize the output calorific value of syngas. A maximum calorific value of $3.84 \mathrm{MJ} / \mathrm{Nm}^{3}$ was obtained using the downdraft reactor with $13 \mathrm{~mm}$ particle size and maximum air flow supply. The verification of the optimum operating point under these conditions showed that such operating conditions favored the production of a gas with high calorific value.
\end{abstract}

Keywords: biomass gasification; calorific value; thermochemical conversion; optimization; syngas 


\section{INTRODUCCIÓN}

Los biocombustibles han atraído mucha atención en los últimos decenios debido al agotamiento del petróleo crudo y el aumento de las emisiones de dióxido de carbono procedentes de la quema de combustibles fósiles. Sin embargo, la sostenibilidad de los biocombustibles de primera generación (etanol de caña de azúcar, etanol a base de almidón o 'maíz', y de biodiesel a partir de aceites vegetales o grasas animales) se ha enfrentado a fuertes críticas porque tales biocombustibles podrían poner en peligro la producción de alimentos (Eisentraut, 2010). Por lo tanto, los biocombustibles de segunda generación producidos a partir de biomasa lignocelulósica, también llamados biocombustibles lignocelulósicos, tienden a ser una mejor opción, ya que no compiten con la producción de alimentos y hay abundante materia prima disponible (Perlack et al., 2005). En este trabajo se evalúan como combustibles de alimentación al proceso carbón vegetal y el cuesco de la palma africana en representación de los combustibles de segunda generación, este último residuo agrícola puede emplearse como combustible debido a la presencia de moléculas celulósicas que contienen energía en sus enlaces y que se libera bajo condiciones de oxidación total o parcial (Mendoza et al., 2012) y se lleva a cabo la comparación de este residuo con carbón vegetal a fin de considerar la conversión de la energía contenida de este combustible en estado sólido en energía contenida en un gas debido a las facilidades de manejo, transporte, almacenamiento, y otras ventajas adicionales a las mencionadas.

Ahora bien, La conversión termoquímica hace referencia a la transformación de un combustible sólido carbonoso a liquido o gaseoso mediante reacciones químicas propiciadas por la adición de calor o un oxidante que propicia una combustión parcial del material aportando la fuente de calor. Estos gases y líquidos tienen ventajas en el transporte, almacenamiento, combustión, flexibilidad en la producción y comercialización. Cuyas propiedades son relativas en gran medida al método de conversión, combustible usado y condiciones del proceso (Bridgwater y Grassi, 1991). La gasificación es uno de los procesos versátiles de conversión termoquímica de mayor antigüedad mediante el cual se produce una mezcla de gas de $\mathrm{H} 2, \mathrm{CO}_{\text {y }} \mathrm{CH}_{4}$, donde las proporciones están determinadas por el uso de aire, oxígeno o vapor de agua como agente oxidante. Uno de los objetivos de la investigación en gasificación es evaluar diferentes diseños de gasificadores de biomasa para generar gas rico en monóxido de carbono e hidrógeno y con bajos contenidos de alquitrán y otras impurezas. Los diseños típicos de gasificador incluyen: lecho fluidizado burbujeante o circulante, un flujo ciclónico o arrastrado y sistemas de lecho fijo ascendente o descendente (Patil et al., 2011). Una variedad de gasificadores de biomasa se han desarrollado a lo largo de los tiempos como modificaciones a los existentes a fin de optimizar el rendimiento de los mismos. La diferenciación se basa en la disposición o apoyo de la biomasa en el reactor, la dirección del flujo tanto de la biomasa y el oxidante, y la forma se suministra calor al reactor (Couto et al., 2013). El gas energético producido en un reactor es una mezcla de especies de gases combustibles rica en hidrógeno, monóxido y dióxido de carbono cuyas proporciones varían de acuerdo al rendimiento del sistema (Dogru et al., 2002; Sheth y Babu, 2009; Olgun et al., 2011). En este trabajo se emplea un gasificador de lecho fijo para llevar a cabo las pruebas en el cual se evalúan dos disposiciones: updraft y downdraft que aluden simplemente al desplazamiento del lecho al interior del reactor.

Este proceso no necesita fuentes externas de calor para mantener la temperatura de gasificación y para mantener las reacciones dentro del proceso. Como resultado, los equipos y requisitos de funcionamiento de la gasificación de biomasa son relativamente menores cuando se usa aire como el agente gasificante. El valor calorífico del gas de síntesis producido por la gasificación con aire es, obviamente, menor que la de oxígeno puro o gasificación de vapor (Pengmei et al., 2007; Umeki et al., 2010; Lucas C, 2004). El valor calorífico del gas de síntesis producto de la gasificación empleando aire como agente gasificante es generalmente inferior a $6 \mathrm{MJ} / \mathrm{Nm} 3$, lo que limita su aplicación en algunos campos industriales (Puig-Arnavat et al., 2010). El aprovechamiento energético de la biomasa lignocelulósica presenta importantes beneficios sociales, económicos y medioambientales dado que la gasificación de estos residuos genera un gas apto para su empleo en motores, por ejemplo (Hernández et al., 2004).

En este caso, se empleó como agente gasificante aire para evaluar el rendimiento del proceso y lograr la optimización de los factores que influyen en el poder calorífico del gas obtenido del proceso, cabe mencionar que fueron seleccionados sólo 4 factores puesto que eran estos los de interés inicial dentro de este trabajo; entonces bien, este estudio se centra en la conversión de biomasa en gas de síntesis o syngas mediante el proceso de micro-gasificación, y el cual ha mostrado una serie de altibajos desde su primera aparición. Haciendo uso de un diseño de experimento como herramienta estadística para la evaluación de la significancia de las cuatro variables de interés en este trabajo y su interacción (granulometría, velocidad del aire, contenido de biomasa y disposición del reactor) en el contenido energético del gas de salida obtenido del proceso se optimizaron dichas variables en pro de maximizar el poder calorífico del syngas. Los resultados documentados en este trabajo son pues de naturaleza experimental y estadística. 


\section{DISEÑO EXPERIMENTAL}

Los dos tipos de biomasa analizados en este trabajo fueron carbón y cuesco de palma africana debido a las similitudes de tamaños de grano para ambos casos. Para obtener el tamaño de grano deseado para las pruebas correspondientes, se llevó a cabo un tamizado previo con tamices de especificaciones de tamaño de 8 y $13 \mathrm{~mm}$. Fue necesario previo al tamizado un proceso de trituración para el carbón a utilizar en la prueba hasta conseguir un tamaño de partícula similar al cuesco.

Tabla 1: Propiedades del cuesco de palma africana

Análisis elemental (base seca)

\begin{tabular}{|c|c|c|c|c|c|c|c|}
\hline & a & $b$ & C & $\mathrm{d}$ & $\mathrm{e}$ & $f$ & Media \\
\hline C & 51.1 & 47.6 & 50.8 & 53.8 & 52.4 & 48.6 & 50.7 \\
\hline $\mathrm{H}$ & 7.0 & 5.2 & 6.7 & 7.2 & 6.3 & 5.2 & 6.3 \\
\hline $\mathrm{N}$ & 1.9 & 0.2 & 1.3 & 0.0 & 0.6 & 0.1 & 0.7 \\
\hline$S$ & - & - & 0.1 & 0.5 & 0.2 & 0.2 & 0.2 \\
\hline 0 & 33.3 & 38.9 & 36.0 & 36.3 & 37.3 & 43.9 & 37.6 \\
\hline
\end{tabular}

Análisis próximo
\begin{tabular}{|l|c|c|c|c|c|c|c|}
\hline CF & $\mathrm{a}$ & $\mathrm{b}$ & $\mathrm{c}$ & $\mathrm{d}$ & $\mathrm{e}$ & $\mathrm{f}$ & Media \\
\hline MV & 13.8 & 17.4 & 18.8 & 18.4 & 19.9 & 19.7 & 18.0 \\
\hline CZ & 74.3 & 67.1 & 71.1 & 73.7 & 73.9 & 67.2 & 71.2 \\
\hline
\end{tabular}

En la tabla 1 se muestran las propiedades del cuesco de palma africana objeto de estudio de esta investigación, información recopilada de los autores: a (Hoseinzadeh Hesas et al., 2013), b (Abd Rashid et al., 2014), c (Ninduangdee y Kuprianov, 2013), d (Yan et al., 2005), e (Husain et al., 2002), f (Abnisa et al., 2011), y en la tabla 2 una aproximación de las propiedades del carbón vegetal resultado de la investigación de (Jiménez, Luisa et al., 2006) sobre carbonizados de origen vegetal (COV) para la generación de antroposoles a partir de madera de Eucalyptus globullus con diferentes condiciones de carbonización (temperatura de carbonización entre $350-550^{\circ} \mathrm{C}$, velocidad de calentamiento de $1-3.5^{\circ} \mathrm{C} / \mathrm{h}$ y tiempo de residencia de 60-120min).

Tabla 2: Propiedades del carbonizado de un material de partida (Adaptada de Jiménez, et al., 2006)

Análisis elemental
\begin{tabular}{|l|c|c|c|c|c|}
\hline \multicolumn{1}{c}{ COV2 } & COV6 & COV9 & COV12 & COV 19 \\
\hline $\mathrm{H}$ & 72.24 & 76.15 & 79.68 & 74.37 & 81.98 \\
\hline $\mathrm{N}$ & 4.85 & 4.69 & 4.35 & 4.73 & 3.51 \\
\hline $\mathrm{O}$ & 0.02 & 0.01 & 0.06 & 0.04 & 0.03 \\
\hline
\end{tabular}

\begin{tabular}{|c|c|c|c|c|c|}
\hline & COV2 & Cov6 & COV9 & COV12 & COV19 \\
\hline MV & 39.12 & 45.01 & 31.45 & 40.46 & 24.05 \\
\hline CF & 57.97 & 51.8 & 66.51 & 57.07 & 73.86 \\
\hline $\mathrm{CZ}$ & 0.26 & 0.31 & 0.23 & 0.21 & 0.27 \\
\hline
\end{tabular}

Un diseño de experimentos fraccionado $2^{4-1}$ con 4 puntos centrales fue usado para estudiar la influencia de las cuatro variables y sus interacciones en la producción de gas de síntesis mediante la gasificación de biomasa residual proveniente del sector agroindustrial de la Región Caribe. El diseño de experimento resultó en 12 corridas experimentales ( 8 corridas factoriales y 4 puntos centrales), donde cada una de las variables posee 3 niveles: $-1,0$ y 1 como se muestra en la Tabla 3.

Tabla 3: Niveles de los factores

\begin{tabular}{|c|l|c|c|c|}
\hline \multicolumn{2}{|c|}{ Variables } & \multicolumn{3}{|c|}{ Niveles } \\
\cline { 3 - 5 } & -1 & 0 & 1 \\
\hline A & Granulometría $(\mathrm{mm})$ & 8 & 10.5 & 13 \\
\hline B & Velocidad del aire $(\mathrm{m} / \mathrm{s})$ & 0.8 & 1.1 & 1.4 \\
\hline C & Contenido de biomasa $(\%)$ & 0 & 50 & 100 \\
\hline D & Disposición del reactor & Up & - & Down \\
\hline
\end{tabular}

Los factores estudiados en este trabajo corresponden a la granulometría o tamaño de partícula de la biomasa en milímetros, la velocidad del aire inyectado al proceso como agente gasificante en $\mathrm{m} / \mathrm{s}$, el contenido de biomasa (un porcentaje del 100\% representa alimentación con cuesco al reactor, un porcentaje de 0\% 
corresponde a un suministro total de carbón vegetal y $50 \%$ representa un $50 \%$ de carbón y $50 \%$ de cuesco) y el tipo de reactor (updraft representa un lecho ascendente y downdraft representa un lecho descendente).

El orden experimental fue aleatorizado debido al tiempo implicado para cada ejecución (aproximadamente 1 hora era consumida entre el proceso como tal, carga y descarga del reactor), esto para evitar que el uso continuo y la temperatura del reactor afectaran a la variable de salida. La Tabla 3 muestra los niveles de los factores analizados en el experimento, se observa que la variable $D$ no es continua, por lo tanto fue seleccionado un número par de puntos centrales para que de esta manera se pudieran distribuir estos puntos a los dos niveles (2 corridas para la disposición updraft y 2 corridas para la disposición downdraft). El nivel de significancia seleccionado para la toma de decisiones en todos los análisis estadísticos de este trabajo correspondió a 0.05 .

Un gasificador de lecho fijo a escala piloto se utilizó para todas las pruebas, las dimensiones del reactor eran: $167 \mathrm{~mm}$ de diámetro, altura de $1480 \mathrm{~mm}$ con un conducto para el flujo de aire de $76.2 \mathrm{~mm}$ de diámetro y 400 $\mathrm{mm}$ de longitud, que se muestra en la Figura 1. En una corrida típica, el reactor se cargó con la biomasa de un tamaño de grano específico, se encendió desde la parte superior o la parte inferior dependiendo de la disposición a evaluar y, finalmente se inyectó el flujo de aire. Para la evaluación del poder calorífico del gas obtenido a partir de proceso se tomaron 18 muestras para cada ejecución (un total de 360 muestras se evaluaron incluyendo ejecuciones del diseño inicial y la optimización del proceso) a través de una conexión directa a un cromatógrafo de gases Micro 490-gc Agilent Technologies de tres columnas de gases se analizaron cuantitativamente mediante la aplicación de la norma estándar ASTM E260-96 (2011) D1945-03 de la ASTM (2010) las concentraciones en porcentaje $n / n$ de los gases combustibles obtenidos.

El rendimiento de un gasificador se cuantifica bajo dos parámetros que son: la composición del gas, que influye directamente en su valor energético y la eficiencia de conversión del reactor, que a su vez se ve afectado por varios factores propios del equipo, por lo tanto el pre-tratamiento del combustible sólido, ya sea biomasa, carbón u otro material carbonoso y las propiedades de este, afectan la conversión del reactor.

La composición del gas obtenido a partir de un sistema de gasificación depende de la composición del combustible y sus cualidades, el agente oxidante, la relación agente/combustible, la presión y temperatura de funcionamiento. Y aun cuando la composición del gas combustible obtenido puede variar considerablemente en cada reactor de acuerdo al combustible y las relaciones utilizadas de oxidante, la mezcla de gases de síntesis mantiene sus componentes combustibles que son, hidrógeno, monóxido de carbono, metano e incombustibles, dióxido de carbono y nitrógeno. En este trabajo el rendimiento de operación del equipo de gasificación se evaluó a través del valor energético del gas de síntesis obtenido del proceso.

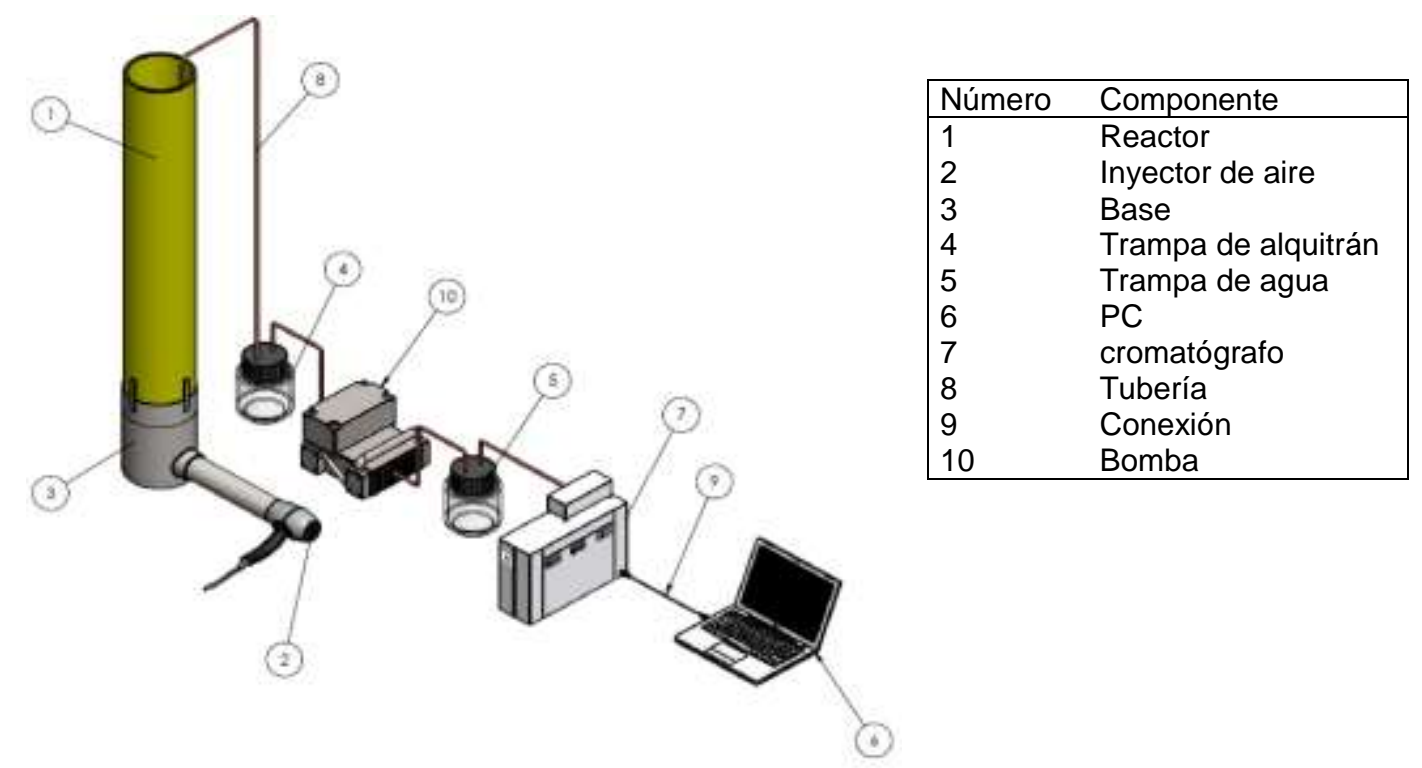

Fig.1: Gasificador de lecho fijo a escala piloto

\section{RESULTADOS Y DISCUSIÓN}

En la tabla 4 se muestra un diseño experimental $2^{4-1}$ inicial para el estudio del efecto de las cuatro variables en el poder calorífico del gas de síntesis a partir de la gasificación de biomasa; las cuatro variables: granulometría, velocidad del aire, contenido de biomasa y disposición del reactor mostraron que bajo estas diferentes combinaciones, el poder calorífico varía entre 0.25 - 4.94MJ / Nm3. 
Tabla 4: Matriz experimental inicial con la variable de salida

\begin{tabular}{|c|c|c|c|c|c|c|}
\hline Corrida & Granulometría & $\begin{array}{c}\text { Velocidad } \\
\text { del aire }\end{array}$ & $\begin{array}{c}\text { Contenido } \\
\text { de biomasa }\end{array}$ & \multicolumn{2}{|c|}{ Reactor } & \multicolumn{2}{|c|}{$\mathrm{PCI}(\mathrm{KJ} / \mathrm{kg} ; \mathrm{Mj} / \mathrm{Nm} 3)$} \\
\hline 1 & 8 & 0.8 & 0 & Up & -2150.36 & 2.763 \\
\hline 4 & 13 & 0.8 & 0 & Down & -4047.6 & 4.942 \\
\hline 3 & 8 & 1.4 & 0 & Down & -1431.02 & 1.821 \\
\hline 2 & 13 & 1.4 & 0 & Up & -1257.56 & 2.282 \\
\hline 8 & 8 & 0.8 & 100 & Down & -955.469 & 1.284 \\
\hline 6 & 13 & 0.8 & 100 & Up & -885.933 & 1.187 \\
\hline 5 & 8 & 1.4 & 100 & Up & -112.181 & 0.255 \\
\hline 7 & 13 & 1.4 & 100 & Down & -1317.09 & 1.751 \\
\hline
\end{tabular}

La selección de la matriz experimental anterior es motivada a manera de filtro o tamiz de factores con el fin de observar la importancia de cada uno de estos y la posible eliminación de algunos de ellos en el experimento como es el caso nuestro.

La Tabla 5 muestra el análisis de la varianza (ANOVA). Este análisis muestra que los principales factores que corresponden al tamaño de las partículas, velocidad del aire y el contenido de biomasa resultan ser los más significativos en el diseño propuesto aun cuando solo la variable correspondiente al contenido de biomasa resultó con un p-value menor a la significancia evaluada en este trabajo (5\%). El factor $D$ fué excluido en análisis posteriores debido a que resultó ser el más alejado. La figura 2 corresponde a la verificación de los supuestos de normalidad e independencia de los datos para esta matriz experimental inicial.

Tabla 5: Análisis de la varianza (ANOVA)

\begin{tabular}{|l|c|c|c|c|c|}
\hline \multicolumn{1}{|c|}{ Fuente } & $\begin{array}{c}\text { Suma de } \\
\text { cuadrados }\end{array}$ & Gdl & $\begin{array}{c}\text { Cuadrado } \\
\text { medio }\end{array}$ & F-Crítico & P-Value \\
\hline A:Granulometría & 2.039 & 1 & 2.039 & 4.290 & 0.130 \\
\hline B:Velocidad del aire & 2.067 & 1 & 2.067 & 4.350 & 0.128 \\
\hline C:Contenido de biomasa & 6.718 & 1 & 6.718 & 14.150 & 0.033 \\
\hline D:Disposición & 1.370 & 1 & 1.370 & 2.890 & 0.188 \\
\hline Error total & 1.424 & 3 & 0.475 & & \\
\hline Total (corr.) & 13.619 & 7 & & & \\
\hline
\end{tabular}
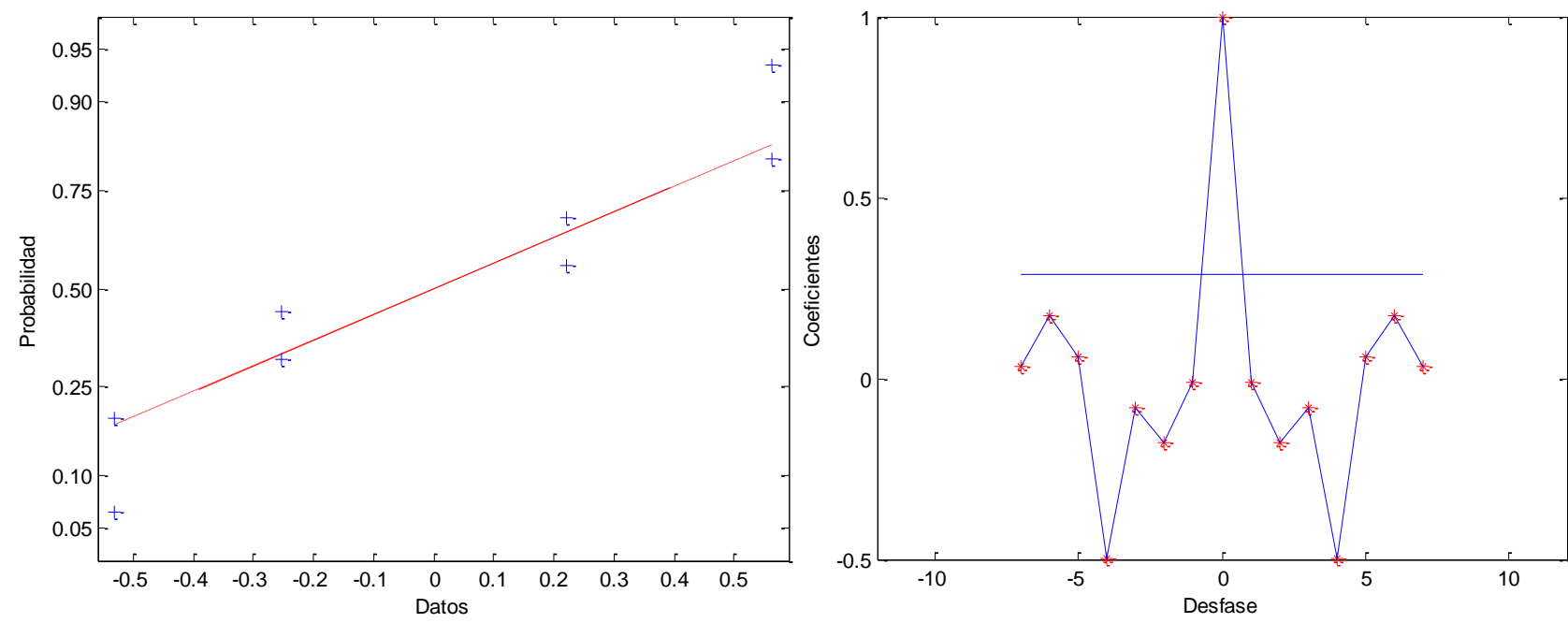

Fig. 2: (a) Gráfico de probabilidad normal. (b) Gráfico de Independencia

Dados los resultados anteriores, a continuación se muestran algunas corridas adicionales llevadas a cabo para consolidar el experimento y tener suficiente apoyo estadístico para la construcción de un modelo de regresión que describa y se ajuste a los datos teniendo en cuenta que el factor correspondiente a la disposición del reactor y el cual no es significativo puede mantenerse constante. Debido a que no era posible valerse de un ascenso máximo para optimizar la variable de salida (poder calorífico del gas) por reestricciones impuestas por el mismo proceso, esta fué la alternativa más viable.

Teniendo en cuenta que la salida de más alto poder calorífico (PCI) se obtuvo con el factor correspondiente al tipo de reactor en la disposición downdraft. en el siguiente análisis esta variable se mantuvo constante. 
Dado que algunas de las corridas que se muestran en los experimentos de la matriz de la Tabla 4 se llevaron a cabo con dicha disposición. se completan las faltantes con este nivel como se muestra en la Tabla 6 . Esto teniendo en cuenta las limitaciones de costo, equipos y tiempo de operación. La tabla 6 contiene la nueva matriz experimental con un nuevo diseño con 3 factores y 4 puntos centrales para evaluar curvatura en el experimento $\left(2^{3}, n_{c}=4\right)$. Los factores de estudio en esta etapa fueron el tamaño de partícula o granulometría, velocidad del aire y el contenido de biomasa y la variable de salida el poder calorífico del gas de síntesis obtenido.

Tabla 6: Matriz de optimización con la variable de salida

\begin{tabular}{|c|c|c|c|c|c|}
\hline Corrida & Granulometría & $\begin{array}{c}\text { Velocidad } \\
\text { del aire }\end{array}$ & $\begin{array}{c}\text { Contenido } \\
\text { de } \\
\text { biomasa }\end{array}$ & \multicolumn{2}{|c|}{$\begin{array}{c}\mathrm{PCl}(\mathrm{KJ} / \mathrm{kg} ; \\
\mathrm{Mj} / \mathrm{Nm} 3)\end{array}$} \\
\hline 1 & 8 & 0.8 & 0 & -609.29 & 0.812 \\
\hline 4 & 13 & 0.8 & 0 & -4047.60 & 4.942 \\
\hline 3 & 8 & 1.4 & 0 & -1431.02 & 1.821 \\
\hline 2 & 13 & 1.4 & 0 & -1601.76 & 2.006 \\
\hline 8 & 8 & 0.8 & 100 & -955.47 & 1.284 \\
\hline 6 & 13 & 0.8 & 100 & -957.61 & 1.271 \\
\hline 5 & 8 & 1.4 & 100 & -822.61 & 1.079 \\
\hline 7 & 13 & 1.4 & 100 & -1317.09 & 1.751 \\
\hline 9 & 10.5 & 1.1 & 50 & -936.575 & 1.255 \\
\hline 12 & 10.5 & 1.1 & 50 & -1051.16 & 1.405 \\
\hline 11 & 10.5 & 1.1 & 50 & -897.78 & 1.200 \\
\hline 10 & 10.5 & 1.1 & 50 & -673.34 & 0.900 \\
\hline
\end{tabular}

La figura 3 corresponde a la probabilidad normal de los efectos del nuevo diseño y se observa que las interacciones de segundo orden $B C$ y $A B$ así como la variable principal $B$ (velocidad del aire) no son significativas, razón por la cual fueron excluidas del modelo de regresión y los análisis subsecuentes. La tabla 7 contiene el análisis de varianza para los factores de interés; se puede observar que el factor B correspondiente a la velocidad del aire no resulta ser significativo por lo cual puede ser excluido del modelo de regresión y las interacciones $A B$ y $B C$ aun cuando en la tabla anova poseen $p$-value menores al nivel de significancia ( $5 \%$ ) son valores grandes en comparación con A, C, AC y la cuadratura; además que en la figura 3 se muestran insignificantes en comparación con los mencionados anteriormente.

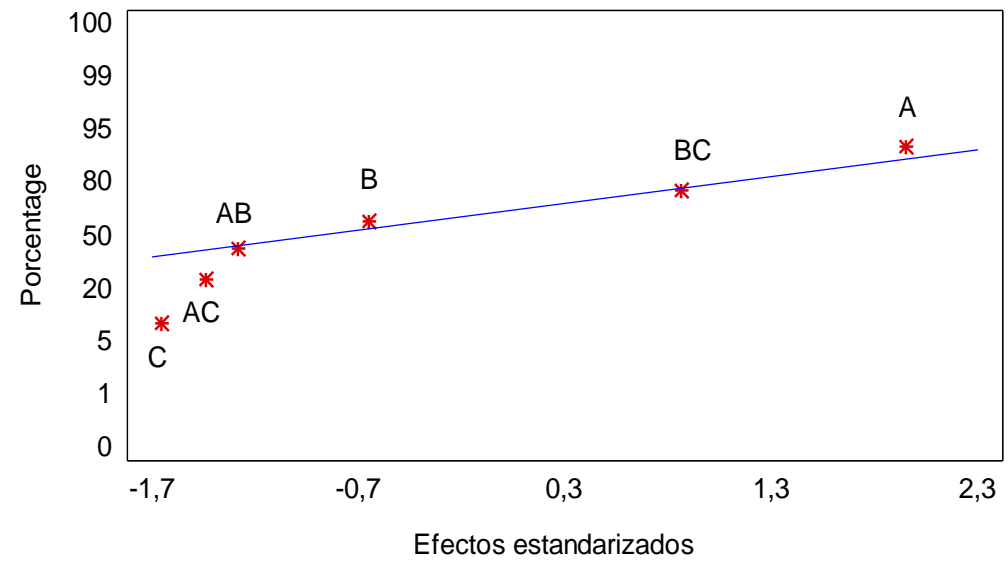

Fig. 3: Gráfico de probabilidad normal de los efectos

Tabla 7: Análisis de la varianza (ANOVA)

\begin{tabular}{|c|c|c|c|c|c|}
\hline Fuente & Suma de cuadrados & Gdl & Cuadrado medio & F-Crítico & $P$-Value \\
\hline A:Granulometría & 3.092 & 1 & 3.092 & 68.900 & 0.004 \\
\hline B:Velocidad del aire & 0.341 & 1 & 0.341 & 7.600 & 0.070 \\
\hline C:Contenido de biomasa & 2.201 & 1 & 2.201 & 49.030 & 0.006 \\
\hline AB & 1.328 & 1 & 1.328 & 29.600 & 0.012 \\
\hline AC & 1.670 & 1 & 1.670 & 37.230 & 0.009 \\
\hline BC & 0.606 & 1 & 0.606 & 13.500 & 0.035 \\
\hline Cuadratura & 3.915 & 2 & 1.957 & 43.620 & 0.006 \\
\hline Error puro & 0.134 & 3 & 0.044 & & \\
\hline Total (corr.) & 13.289 & 11 & & & \\
\hline
\end{tabular}



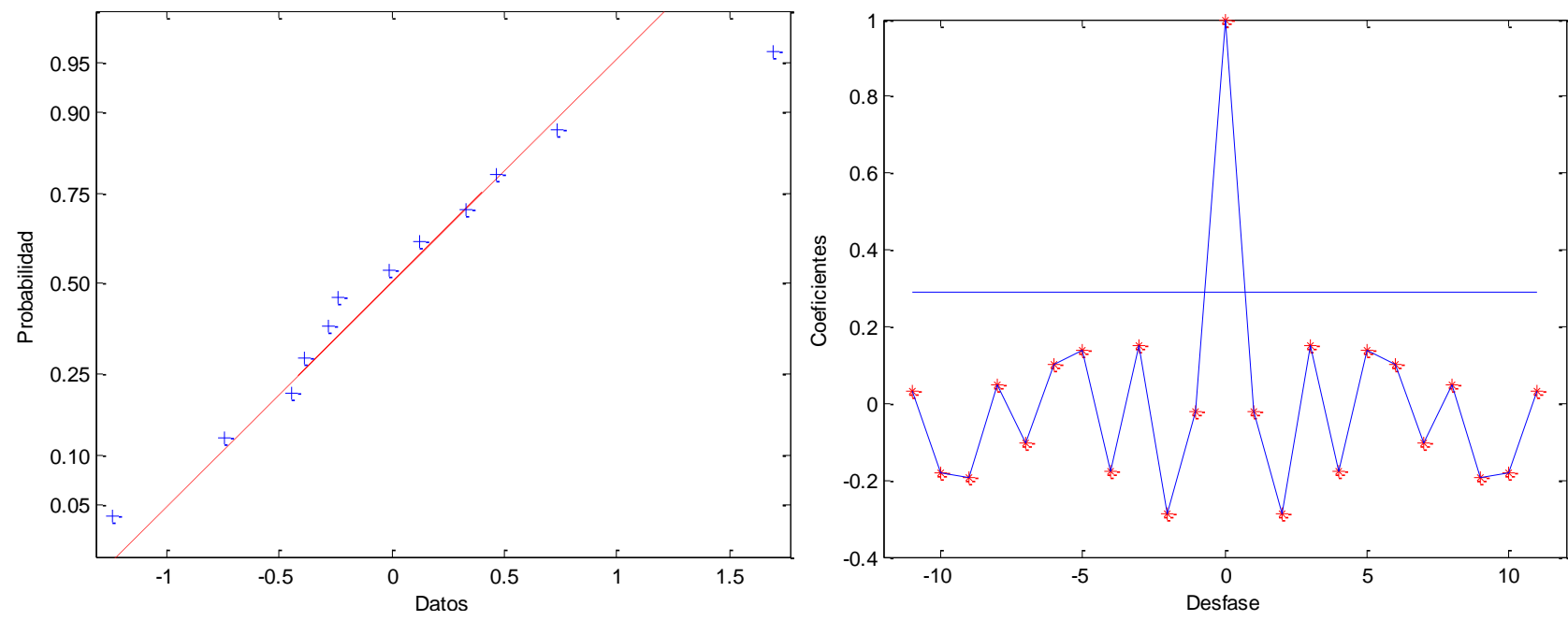

Fig. 4: (a) Gráfico de probabilidad normal. (b) Gráfico de Independencia

La ecuación 1 corresponde al modelo de regresión que se ajusta a los datos. Este modelo es presentado en variables codificadas (1-3) y los factores significativos correspondientes a la granulometría y el contenido de biomasa así como la interacción de los dos factores (AC) son incluidas en el mismo. El modelo propuesto no es jerárquico debido a que para este caso resulta mas conveniente que la ecuación que describe el proceso no incluya el término A independente tal y como lo propone la jerarquía.

$$
P C I=0.939 * C-0.652 * A C+0.506 * A^{2}
$$

Para ajustar el modelo de regresión fue necesaria una codificación en la cual el nivel bajo de las variables fue representado por el número 1, el nivel medio por el número 2 y el más alto con el numero 3 respectivamente; esto, dado que una de las variables de interés (contenido de biomasa) posee como uno de sus niveles el valor cero (0) representando problemas en el ajuste correcto del modelo de regresión. Las ecuaciones de codificación correspondientes a la granulometría y al contenido de biomasa son las que se muestran a continuación:

$$
\begin{aligned}
& A_{\text {Codificado }}=0.4 * A_{\text {Natural }}-2.2 \\
& C_{\text {Codificado }}=0.02 * C_{\text {Natural }}+1
\end{aligned}
$$

Además de esto, la verificación de la idoneidad del modelo y la cual implica el cumplimiento de tres criterios primarios: estadísticos de la regresión (Coeficiente R2), significancia del modelo y significancia de los factores incluidos en el modelo se encuentran dentro de este trabajo. La Tabla 8 muestra que el modelo propuesto explica el $87.1 \%$ de la variabilidad en la variable de salida, lo cual resulta un gran valor considerando los valores incluidos en el mismo.

Las Tablas 9 y 10 muestran la verificación de la significancia del modelo y los factores que hacen parte de él, y el cual efectivamente se ajusta a los datos. Se observa en la Tabla 9 que con un p-value de 0.003 , el modelo seleccionado se ajusta al comportamiento de los datos en cuestión; por otro lado, la tabla 10 muestra que los factores incluidos en el modelo son significativos debido a que los $p$-value para los tres casos evaluados fueron menores a 0.05 .

Tabla 8: Estadísticos de la regresión

\begin{tabular}{|l|c|}
\hline Coeficiente de correlación múltiple & 0.933 \\
\hline Coeficiente de determinación $\mathrm{R}^{\wedge} 2$ & 0.871 \\
\hline $\mathrm{R}^{\wedge} 2$ Ajustado & 0.692 \\
\hline Error estándar & 0.894 \\
\hline Observaciones & 10 \\
\hline
\end{tabular}


Tabla 9: Significancia del modelo

\begin{tabular}{|c|c|c|c|c|c|}
\hline Fuente & Gdl & $\begin{array}{c}\text { Suma de } \\
\text { cuadrados }\end{array}$ & $\begin{array}{c}\text { Cuadrado } \\
\text { medio }\end{array}$ & $\begin{array}{c}\text { F- } \\
\text { Crítico }\end{array}$ & P-Value \\
\hline Regresión & 3 & 37.868 & 12.623 & 15.790 & 0.003 \\
\hline Residuos & 7 & 5.596 & 0.799 & & \\
\hline Total & 10 & 43.464 & & & \\
\hline
\end{tabular}

Tabla 10: Significancia de los factores

\begin{tabular}{|c|c|c|c|c|c|c|}
\hline Factor & Coeficiente & $\begin{array}{c}\text { Error } \\
\text { estándar }\end{array}$ & $\begin{array}{c}\text { Estadístico } \\
\mathrm{t}\end{array}$ & $\begin{array}{c}\mathrm{P}- \\
\text { Value }\end{array}$ & $\begin{array}{c}\text { Inferior } \\
95 \%\end{array}$ & $\begin{array}{c}\text { Superior } \\
95 \%\end{array}$ \\
\hline $\mathrm{A}^{2}$ & 0.506 & 0.110 & 4.582 & 0.003 & 0.244 & 0.766 \\
\hline $\mathrm{C}$ & 0.939 & 0.338 & 2.780 & 0.027 & 0.140 & 1.738 \\
\hline $\mathrm{AC}$ & -0.652 & 0.226 & -2.882 & 0.024 & -1.187 & -0.117 \\
\hline
\end{tabular}

La Figura 5 muestra la superficie de respuesta de la variable de salida (en variables codificadas) descrita por el modelo de regresión propuesto previamente, y en el cual se muestra a medida que el tamaño de partícula o granulometría aumenta y el contenido de biomasa decrece, el poder calorífico del gas de salida se incrementa significativamente.

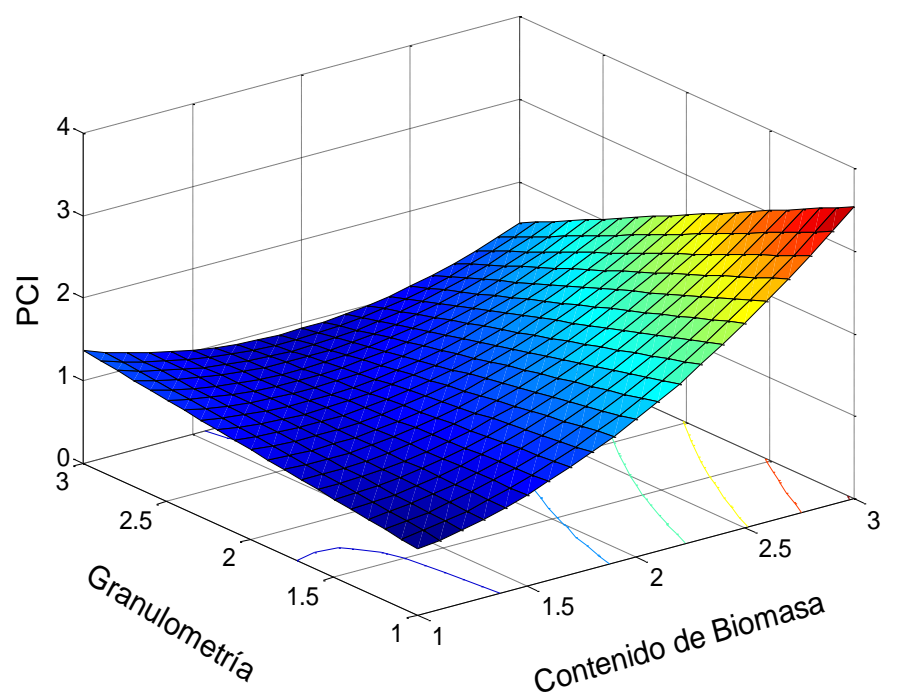

Fig. 5: Gráfico de superficie de respuesta para el Poder Calorífico

La optimización del modelo de regresión que describe el comportamiento de la variable de salida correspondiente al poder calorífico del gas de síntesis analizado muestra que el máximo valor de salida corresponde a 3.54MJ/Nm3 y fue obtenido con el más alto tamaño de partícula y bajo contenido de biomasa; esto es, especificaciones de 13mm para el tamaño de grano y 100\% contenido de carbón. Para corroborar esta optimización, se llevaron a cabo algunas corridas adicionales con las condiciones de granulometría y contenido de biomasas mencionadas. Teniendo que la media de los datos presentados en la tabla 6 corresponden a 3.84MJ/Nm3 y desviación estándar de 0.406, se llevó a cabo una prueba de hipótesis con un intervalo de confianza del $95 \%$ asumiendo como hipótesis nula que la media de los datos era igual a $3.54 \mathrm{MJ} / \mathrm{Nm} 3$ y la hipótesis alterna que la media de los datos era diferente a $3.84 \mathrm{Mj} / \mathrm{Nm} 3$ para lo cual un pvalue de 0.228 fué obtenido y por lo cual la hipótesis nula fue rechazada.

Mientras que la velocidad del aire suministrado al proceso se mostró como un factor no significativo, los valores de la variable de salida evaluada teniendo en cuenta este factor se promediaron y se observó que los valores más altos de poder calorífico fueron obtenidos con este factor en su nivel mas bajo; luego entonces, para la optimización este factor fué mantenido constante en el nivel correspondiente a $0.8 \mathrm{~m} / \mathrm{s}$.

Tabla 11: Réplicas de verificación del punto óptimo de operación

\begin{tabular}{|c|c|}
\hline Replica & $\mathrm{PCl}(\mathrm{MJ} / \mathrm{m} 3)$ \\
\hline 1 & 4.264 \\
\hline 2 & 4.048 \\
\hline 3 & 3.327 \\
\hline 4 & 3.745 \\
\hline
\end{tabular}


El valor del poder calorífico inferior del gas obtenido fue comparado con datos presentados en pruebas de microgasificación de biomasa para utilización en estufas (Raman et al., 2013). En estas pruebas, se utiliza biomasa de cáscara de coco (Forero et al., 2012) que tiene características físicas similares al cuesco de palma utilizado en esta investigación. La eficiencia de conversión (Raman et al., 2013) está entre 27 y 36\%, y para un poder calorífico inferior de $18 \mathrm{MJ} / \mathrm{kg}$ del cuesco de palma, se observa que los valores fluctúan entre 4.8 y 6.4 $\mathrm{MJ} / \mathrm{kg}$. Estos valores están en el mismo orden de magnitud del valor más alto observado en la experimentación que es de $4.05 \mathrm{MJ} / \mathrm{kg}$, el cual fue seleccionado para llevar a cabo el proceso de optimización.

\section{CONCLUSIONES}

Basados en el análisis del diseño experimental y el modelo de predicción propuesto, se encontró que los más altos valores de poder calorífico fueron obtenidos en el reactor de lecho fijo con disposición descendente, alto contenido de carbón, la más alta granulometría y baja velocidad de inyección de aire como agente gasificante donde las cuatro variables variaban desde $8 \mathrm{~mm}-13 \mathrm{~mm}$ para la granulometría, $0.8-1.4 \mathrm{~m} / \mathrm{s}$ para la velocidad del aire, 0-100\% para el contenido de biomasa y updraft - Downdraft para la disposición del reactor. Un valor máximo de poder calorífico de $3.84 \mathrm{MJ} / \mathrm{Nm} 3$ se obtuvo usando la disposición downdraft del reactor, alimentación total de carbón, tamaño de grano de $13 \mathrm{~mm}$ y velocidad del aire de $0.8 \mathrm{~m} / \mathrm{s}$.

La validación de los resultados experimentales (con las condiciones de operación de optimización) fué cercana al valor máximo previsto, lo que indica que el modelo de regresión basado en los datos experimentales se ajusta a los datos analizados en la predicción y optimización propuesta. El modelo estadístico de regresión se precisa solamente para las condiciones específicas de este trabajo, por lo cual puede ser solamente aplicado a los factores específicos con los que se trabajó en este documento. Para un reactor diferente y la evaluación de los mismos factores aquí estudiados los resultados podrían ser diferentes. Teniendo estas limitaciones en cuenta, el modelo desarrollado en este estudio es útil para revelar el efecto de cada término significativo y sus interacciones en la variable de salida pero dentro de los parámetros, factores, condiciones, equipos e instrumentos aquí seleccionados.

\section{AGRADECIMIENTOS}

Este artículo es resultado del proyecto de investigación Implementación de proyectos de I+D (componentes Microalgas, Extractos de plantas y cadena productiva de la sábila, Producción 1.2 Pro-panodiol y Plataforma Informática) para promover el desarrollo y la transferencia tecnológica de cadenas productivas agroindustriales y la implementación de tecnologías de última gene-ración para el procesamiento de biocombustibles en el Departamento del Atlántico, el cual es financiado por el Departamento del Atlántico a través de recursos del Sistema General de Regalías - Fondo de Ciencia, Tecnología e Innovación.

\section{REFERENCIAS}

Abd Rashid, R., Mohd Salleh, H., Hanafi Ani, M., Azhani Yunus, N., Akiyama, T., y Purwanto, H., "Reduction of low grade iron ore pellet using palm kernel shell'. Renewable Energy. Vol 63. 617-623 (2014)

Abnisa, F., Wan Daud, W., Husin, W. y Sahu, J., "Utilization possibilities of palm shell as a source of biomass energy in Malaysia by producing bio-oil in pyrolysis process". Biomass and Bioenergy. Vol 35(5). 1863-1872 (2011)

Bridgwater, A. y Grassi, G., "Biomass Pyrolysis Liquids Upgrading And Utilisation". London and New York: Elsevier Applied Science. (1991)

Couto. N., Rouboa. A., "Influence of the biomass gasification processes on the final composition of syngas". Energy Procedia 36. 596-606 (2013)

Dogru. M., Howarth. C., Akay. G., Keskinler. B. y Malik. A. "Gasification of hazelnut shells in a downdraft gasifier". Energy. 27(5) 415-427 (2002)

Eisentraut, A., "Sustainable production of second-generation biofuels: potential and perspectives in major economies and developing countries". International Energy Agency. (2010)

Forero Núñez, Carlos A., Jochum, Joachim., Sierra Vargas, Fabio E., "Characterization and feasibility of biomass fuel pellets made of Colombian timber, coconut and oil palm residues regarding European standards". Environmental Biotechnology 8 (2) 67-76 (2012) 
Hernández, J.J., Serrano, C., Pérez, J., Horrillo, A.J., "Velocidad de Combustión Laminar del Gas de Gasificación Calculada usando Diferentes Mecanismos de Reacción”. Información Tecnológica. Vol 15(3). 19$22(2004)$

Hoseinzadeh Hesas, R., Arami-Niya, A., Ashri Wan Daud, W. y Sahu, J., "Comparison of oil palm shell-based activated carbons produced by microwave and conventional heating methods using zinc chloride activation". Journal of Analytical and Applied Pyrolysis. Vol 104. 176-184 (2013)

Husain, Z., Zainal, Z. y Abdullah, M., "Analysis of biomass-residue-based cogeneration system in palm oil mills". Biomass and Bioenergy. Vol 24(2). 117-124 (2003)

Jiménez, L.F., Baquero, M.C. y Díaz, J., "Carbonizados de origen vegetal (COV) para la generación de antroposoles. Obtención y caracterización fisicoquímica". Rev.Colomb.Quim. vol.35 no.2 (2006)

Lucas. C., Szewczyk. D., Blasiak. W. y Mochida, S. "High-temperature air and steam gasification of densified biofuels". Biomass and Bioenergy 27 (6). 563-575 (2004)

Mendoza, Jorge M., Bula, Antonio J., Gómez, Rafael D., Corredor, Lesmes A. "Análisis exergético de la gasificación de biomasa". Información tecnológica 23(5), 85-96 (2012)

Ninduangdee, P. y Kuprianov, V. "Study on burning oil palm kernel shell in a conical fluidized-bed combustor using alumina as the bed material". Journal of the Taiwan Institute of Chemical Engineers. Vol 44(6). 10451053 (2013)

Olgun, H., Ozdogan, S. y Yinesor, G. "Results with a bench scale downdraft biomass gasifier for agricultural and forestry residues". Biomass and Bioenergy. 35(1) 572-580 (2011)

Patil, K., Bhoi. P., Huhnke. R. y Bellmer, D. "Biomass downdraft gasifier with internal cyclonic combustion chamber: Design. construction. and experimental results”. Bioresource Technology 102. 6286-6290 (2011)

Pengmei, L., Zhenhong, Y. y Longlong, M. "Hydrogen-rich gas production from biomass air and oxygen/steam gasification in a downdraft gasifier". Renewable Energy (32) 2173-2185 (2007)

Perlack, R., Wright, L., Turhollow, A., Graham, R., Stokes, B., y Erbach, D. "Biomass as feedstock for a bioenergy and bioproducts Industry: the technical feasibility of a billion-ton annual supply". US Depar: Oar Ridge National Laboratory Report ORNL/TM-2005/66 (2005)

Puig-Arnavat, M., Bruno, J.C., Coronas, A. "Review and analysis of biomass gasification models". Renewable and Sustainable Energy Reviews (14) 2841-2851 (2010)

Raman, P., Murali, J., Sakthivadivel, D., Vigneswaran, V.S. "Performance evaluation of three types of forced draft cook stoves using fuel wood and coconut shell'. Biomass and Bioenergy 49, 333-340 (2013)

Sheth, P. y Babu, B. "Experimental studies on producer gas generation from wood waste in a downdraft biomass gasifier”. Bioresource Technology 100(12) 3127-3133 (2009)

Umeki, K., Yamamoto, K., Namioka, T. y Yoshikawa, K. "High temperature steamonly gasification of woody biomass". Applied energy 87 (3). 791-798 (2010)

Yan, R., Yang, H., Chin, T., Tee Liang, D., Chen, H. y Zheng, C. "Influence of temperature on the distribution of gaseous products from pyrolyzing palm oil wastes". Combustion and Flame. Vol 142. (1-2). 24-32 (2005) 